\title{
Clinical outcomes of combined pars plana vitrectomy and trans- scleral 4-point suture fixation of a foldable intraocular lens
}

\author{
Neha Goel ${ }^{1}$
}

Received: 30 September 2017 / Revised: 28 November 2017 / Accepted: 5 December 2017 / Published online: 5 February 2018

(c) The Royal College of Ophthalmologists 2018

\begin{abstract}
Objective: To describe outcomes of combined pars plana vitrectomy (PPV) and trans-scleral 4-point suture fixation of a foldable Akreos AO60 intraocular lens (IOL) in eyes with subluxated or dislocated lens or IOL.

Methods: Retrospective case series of 20 eyes of 18 patients who underwent this procedure with a minimum follow-up of 3 months. Outcome measures included final best-corrected Snellen visual acuity (BCVA), spherical equivalent (SE) and intraoperative and postoperative complications.

Results: Mean age at surgery was 43.65 years. The commonest indication for surgery was post-traumatic subluxated or dislocated cataract. Mean BCVA improved from 6/24 preoperatively to 6/9 at final follow-up ( $p<0.0001)$. Mean SE was 5.69 dioptres preoperatively and -1.32 dioptres postoperatively. There were no intraoperative complications. Postoperative complications included transient vitreous haemorrhage in one eye and cystoid macular oedema in one eye. Postoperative Pentacam analysis confirmed the absence of IOL tilt or decentration in six eyes. Mean follow-up was 8.9 months (range 3-30 months).

Conclusion: Trans-scleral 4-point suture fixation of a foldable Akreos AO60 IOL can be performed safely along with concurrent PPV across varying surgical indications resulting in a substantial improvement in visual acuity with minimal complications.
\end{abstract}

\section{Introduction}

Intraocular lens (IOL) implantation in eyes with insufficient capsular support can be achieved using: anterior chamber IOL (ACIOL), iris-fixated IOL, sutured scleral-fixated posterior chamber IOL (PCIOL) [1] or intrascleral haptic fixation [2, 3]. Each of these techniques comes with its own set of advantages and complications. Several techniques for sutured scleral-fixated IOLs (SFIOL) have been described. Many of them use a rigid polymethylmethacrylate IOL requiring an incision of 6-7 $\mathrm{mm}$.

A subset of these patients may require concomitant pars plana vitrectomy (PPV) for subluxated or dislocated crystalline lens or IOL. These eyes pose an extra challenge because of the lack of vitreous support. Creation of a large incision for IOL may increase the risk of choroidal effusion

Neha Goel

nehadoc@hotmail.com

1 ICARE Eye Hospital and Postgraduate Institute, Noida, UP, India and suprachoroidal haemorrhage [4], in addition to increased astigmatism and longer visual rehabilitation. Currently, there is no foldable PCIOL designed specifically for scleral fixation. Most foldable IOLs do not have eyelets that allow for suture fixation. Tying a suture to the haptic may create a knot or barb that could irritate the uveal tissue, resulting in inflammation. Sutures that are tied to a haptic are prone to slipping off the haptic or may result malposition of the IOL [5].

The Akreos AO60 (Bausch \& Lomb, Rochester, NY) is a hydrophilic acrylic foldable IOL that has a natural build for 4-point fixation because of holes in each haptic. Previous studies have determined the feasibility, safety and efficacy of using this IOL for trans-scleral suture fixation [6-10]. Although SFIOL after PPV as a secondary procedure is common, very few studies have evaluated concurrent PPV and SFIOL [11-13]. This obviates the need for two surgeries and also enables suturing the IOL in the ciliary sulcus without vitreous traction. This study was undertaken to evaluate the clinical outcomes of trans-scleral 4-point suture fixation of Akreos AO60 IOL using polypropylene suture, in eyes requiring concomitant PPV. 


\section{Materials and methods}

A retrospective review of patients who underwent transscleral 4-point suture fixation of a foldable Akreos AO60 IOL along with PPV at a tertiary eye centre between September 2013 and March 2017 was performed. Informed consent was obtained from all patients or guardians. Offlabel status of scleral fixation of Akreos AO60 IOL was explained. The study was conducted in accordance with the tenets of the Declarations of Helsinki and has been approved by the institutional ethics board.

The inclusion criteria were eyes with a deficient capsular support in which PCIOL implantation was not possible and underwent concomitant PPV for subluxated or dislocated crystalline lens or IOL. Only those patients who completed a minimum follow-up of 3 months were enrolled.

Data collected included demographic details, indication for surgery, details of ophthalmic examination (including slit lamp examination, intraocular pressure (IOP) by applanation tonometry and indirect ophthalmoscopy), preoperative and final best-corrected Snellen visual acuity (BCVA), pre-operative and final spherical equivalent (SE), intraoperative and postoperative complications and followup period. Results of postoperative Pentacam (Oculus Inc) when performed were also analysed. Biometry was carried out using contact A-scan ultrasound with the OcuScan RxP Ophthalmic Ultrasound System (Alcon Inc) and using keratometry with a Super KMS 6 (Bausch \& Lomb Inc). The IOL power was calculated using the SRK/T formula.

\section{Surgical technique}

All surgeries were performed by the same surgeon (N.G.). The major surgical steps are depicted in Figs. 1(a-i). Following limited conjunctival peritomy in the nasal and temporal quadrants, an 8-cut Radial Keratotomy marker was used to mark the limbus at two points in the horizontal plane $180^{\circ}$ apart. Two partial thickness $3 \times 3 \mathrm{~mm}$ rectangular limbus-based scleral flaps were made at the 3 and 9o'clock positions. Standard 23-gauge PPV was carried out using Accurus Vitrectomy System (Alcon Inc). Lensectomy or phacofragmentation of the subluxated or dislocated crystalline lens was performed (after enlarging one of the sclerotomies to 20 gauge in case of the latter). Attempts were made to remove all peripheral capsular remnants. In cases with a dislocated IOL, the IOL was lifted using 23gauge forceps and explanted through a superior clear corneal incision created using a 3.2-mm keratome. The superior sclerotomies were plugged. The anterior chamber was entered through a clear corneal incision and stabilised using a viscoelastic after clamping the infusion line. A 9-0 polypropylene (Ethicon Inc) suture with a straight needle was passed through the inferior end of the scleral flap 1.0 $\mathrm{mm}$ posterior to the limbus on the right side, externalized through the superior clear corneal incision, threaded through the inferior haptic of the IOL and then through the superior haptic. A 26-gauge hollow needle was passed through the superior end of the scleral flap $1.0 \mathrm{~mm}$ posterior to the limbus and used to capture the Prolene needle that was re-passed through the corneal incision, using an abexterno technique. The same procedure was carried out on the left-sided flap. After the suture was passed, the IOL was folded with two tying forceps and inserted. Traction was then placed on the sutures to remove the slack and centre the IOL. The needles were cut off the suture and the suture ends tied carefully. The knots were buried by carefully rotating them into the 26-gauge needle entry site. The corneal wound was hydrated and 23-gauge cannulas removed. All the incision sites were checked for leakage and sutured if necessary. The nasal and temporal conjunctival incisions were sutured with 8-0 polyglactin (Vicryl, Ethicon Inc) suture. Standard postoperative medications were prescribed.

Patients were followed up on the first postoperative day, first postoperative week and subsequently individualised on a case-to-case basis. The main outcome measures were final BCVA and SE and intraoperative and postoperative complications. Data were entered in a Microsoft Excel and analysed using SPSS version 16.1. The decimal BCVA value was converted to the logarithm of the Minimum Angle of Resolution (logMAR). Counting fingers were converted to 2.0, hand movements were converted to 2.3, and light perception was converted to 3.0. Results are expressed as mean \pm SD. $p<0.05$ was considered statistically significant. Statistical evaluations were based on nonparametric methods. The Wilcoxon signed-rank test was used for related samples.

\section{Results}

In all, 20 eyes of 18 patients were enrolled in this study. The mean age at surgery was $43.65 \pm 16.02$ years (range 15-73). In all, 18 eyes were of male patients, while 2 eyes were of female patients. Right and left eyes were equally distributed. The mean follow-up was 8.9 months, ranging from 3 to 30 months. The most common indication for concomitant PPV and SFIOL was post-traumatic subluxated or dislocated cataract in 11 eyes (55\%). This was followed by ectopia lentis and subluxated or dislocated IOL in four eyes each $(20 \%)$. One patient had a displaced iris claw lens.

Table 1 shows the individual details of each patient. Both patients with bilateral ectopia lentis also had amblyopia. Eight eyes (40\%) also had glaucoma, of which six were post-traumatic and two had juvenile open angle glaucoma. All eyes had IOP controlled on topical antiglaucoma medications; one eye with post-traumatic glaucoma had 


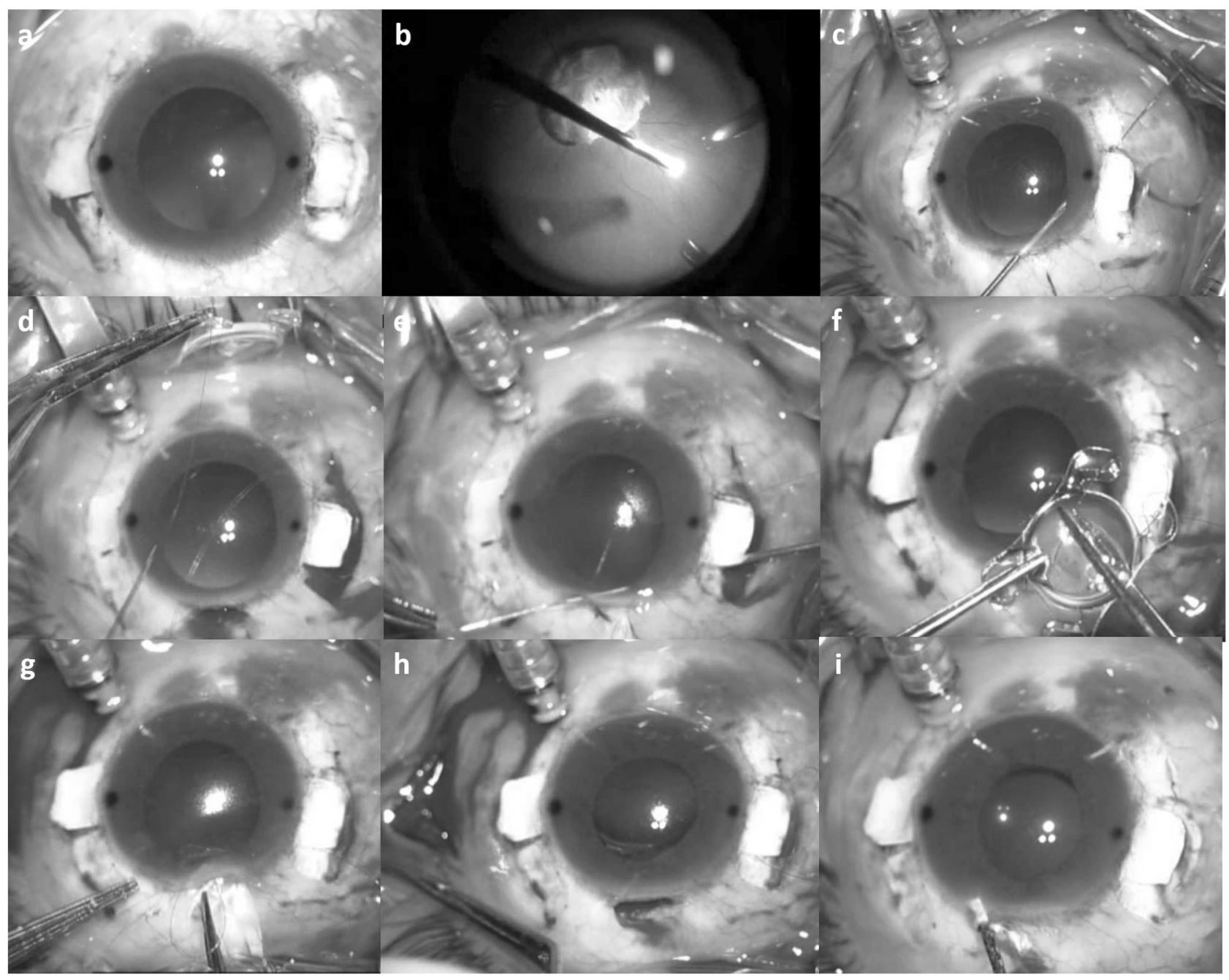

Fig. 1 Intraoperative photographs of case 7 illustrating the major steps of the surgical procedure. a Partial thickness rectangular limbus-based scleral flaps measuring $3 \times 3 \mathrm{~mm}$ are made at 3 and 9 o'clock positions. b Twenty-three gauge pars plana vitrectomy is carried out with phacofragmentation of the lens. c 9-0 polypropylene suture is passed under the nasal scleral flap inferiorly and externalized using a 26gauge needle through a superior clear corneal incision made with 3.2 $\mathrm{mm}$ keratome $\mathbf{d}$ The polypropylene suture is threaded first through the inferior eyelet of the Akreos AO60 IOL and then through the superior eyelet. e A 26-gauge needle is passed under the scleral flap superiorly to retrieve the polypropylene straight needle passed through the superior corneal incision, using an ab-externo technique. f A similar manoeuvre is carried out on the temporal side. $g$ The IOL is inserted through the superior corneal incision after being folded using forceps. $\mathbf{h}$ Traction is placed on the nasal and temporal sutures to remove the slack and centre the IOL, following which they are tied to each other and the knot buried by rotation. $\mathbf{i}$ The superior incision is hydrated and left sutureless undergone trabeculectomy 2 months back (patient number 10). Slit lamp examination showed traumatic mydriasis in eight patients $(40 \%)$. Posterior segment examination was within normal limits in all eyes except patient number 1 who showed macular dystrophy.

All eyes in the series had successful scleral fixation of the IOL and no intraoperative complications occurred. Mean BCVA improved from $6 / 24(0.65 \pm 0.2 \log$ MAR $)$ at presentation to $6 / 9(0.23 \pm 0.2 \log$ MAR) at final follow-up ( $p$ $<0.0001)$. All patients had an improvement in BCVA; none of the eyes had an unchanged or decreased BCVA postoperatively. In all, 13 eyes (65\%) achieved a BCVA of $\geq 6 / 9$ (0.2 $\log$ MAR). Mean SE was 5.69 dioptres preoperatively and -1.32 dioptres postoperatively; the mean change was 8.16 dioptres. Compared to a pre-operative mean IOP of $16.5 \pm 3.8 \mathrm{~mm} \mathrm{Hg}$, IOP was $17.2 \pm 3.1 \mathrm{~mm} \mathrm{Hg}$ at 1 week $(p$ $=0.36)$ and $15 \pm 3.3 \mathrm{~mm} \mathrm{Hg}$ at the final follow-up ( $p=$ 0.10). Scheimpflug imaging using Pentacam was performed in six eyes (30\%) postoperatively. The optic was considered not tilted when the reference line along the iris and the IOL optic were parallel [14]. None of these eyes showed IOL decentration or tilt (Fig. 2). One patient developed transient vitreous haemorrhage for 2 weeks, while one had cystoid macular edema (CME) at 3 weeks postoperatively that resolved within a month on topical Nepafenac $0.3 \%$. No cases of hyphema, corneal oedema, wound leakage, IOL 


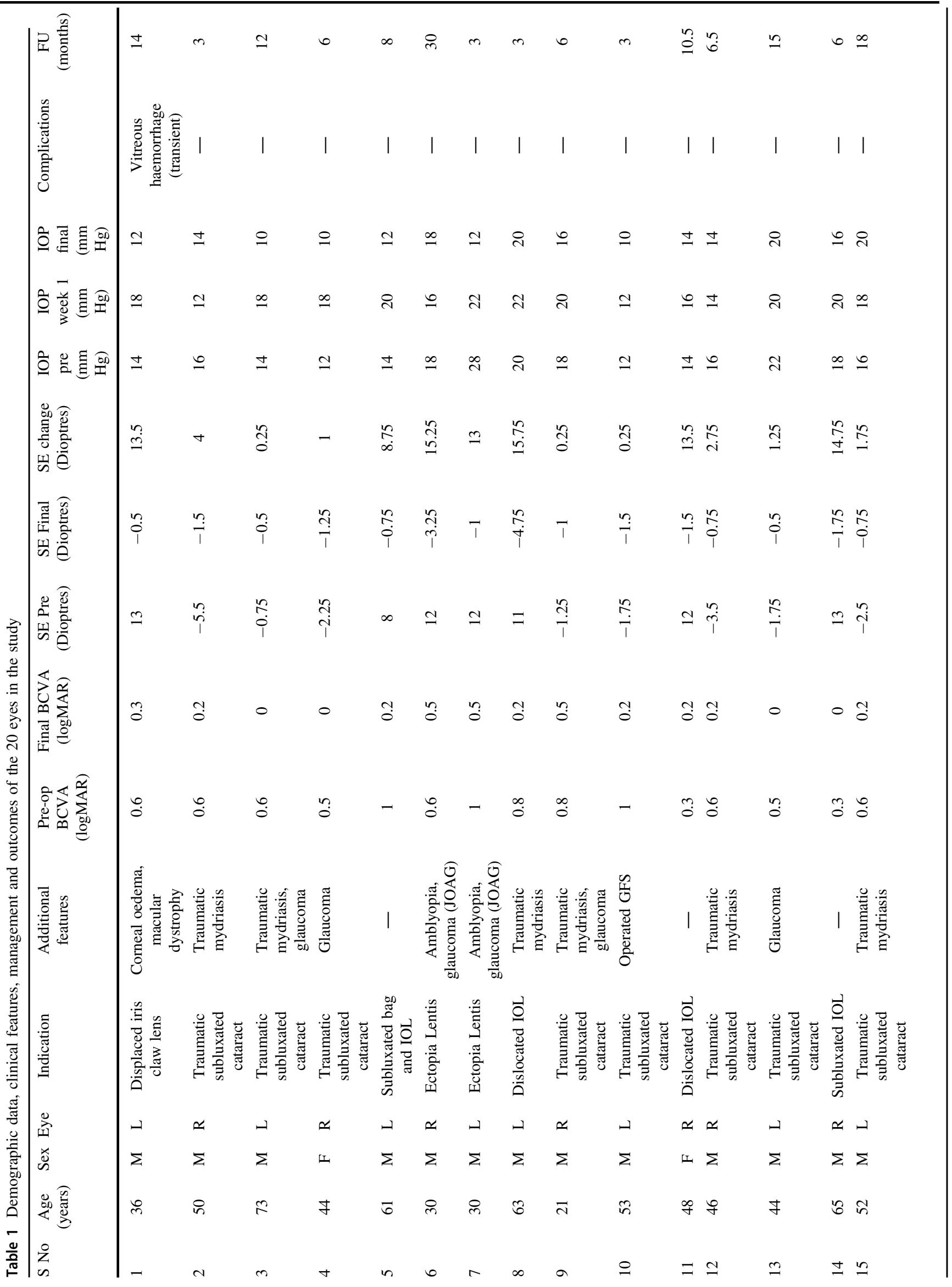




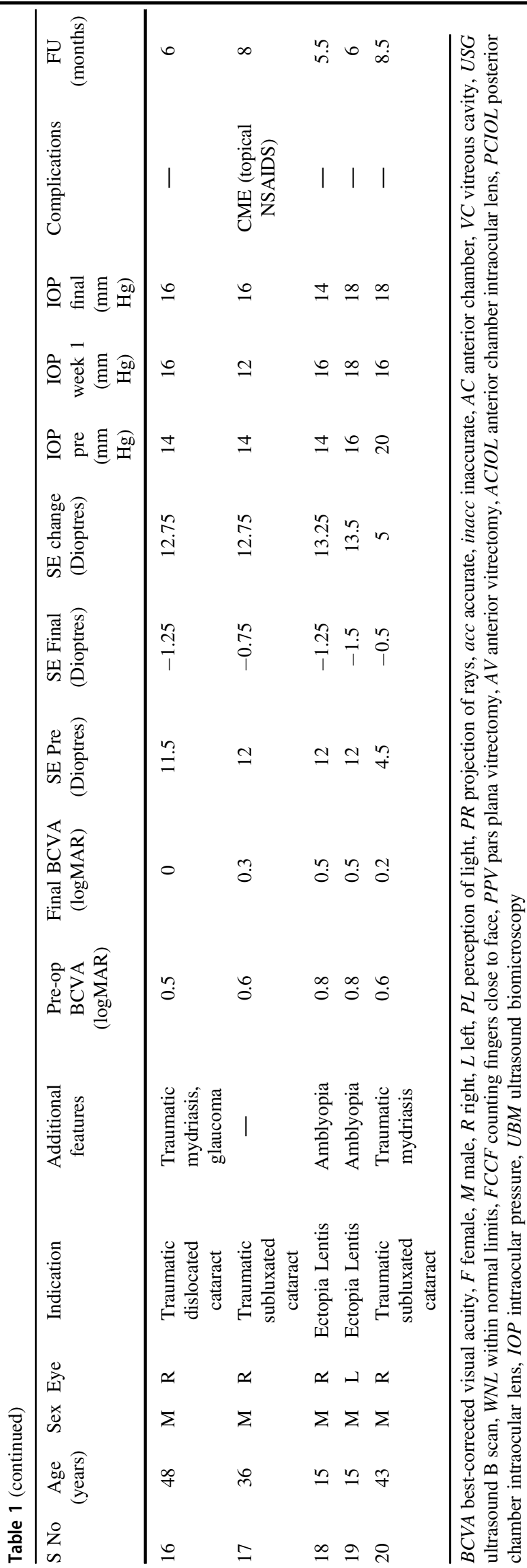
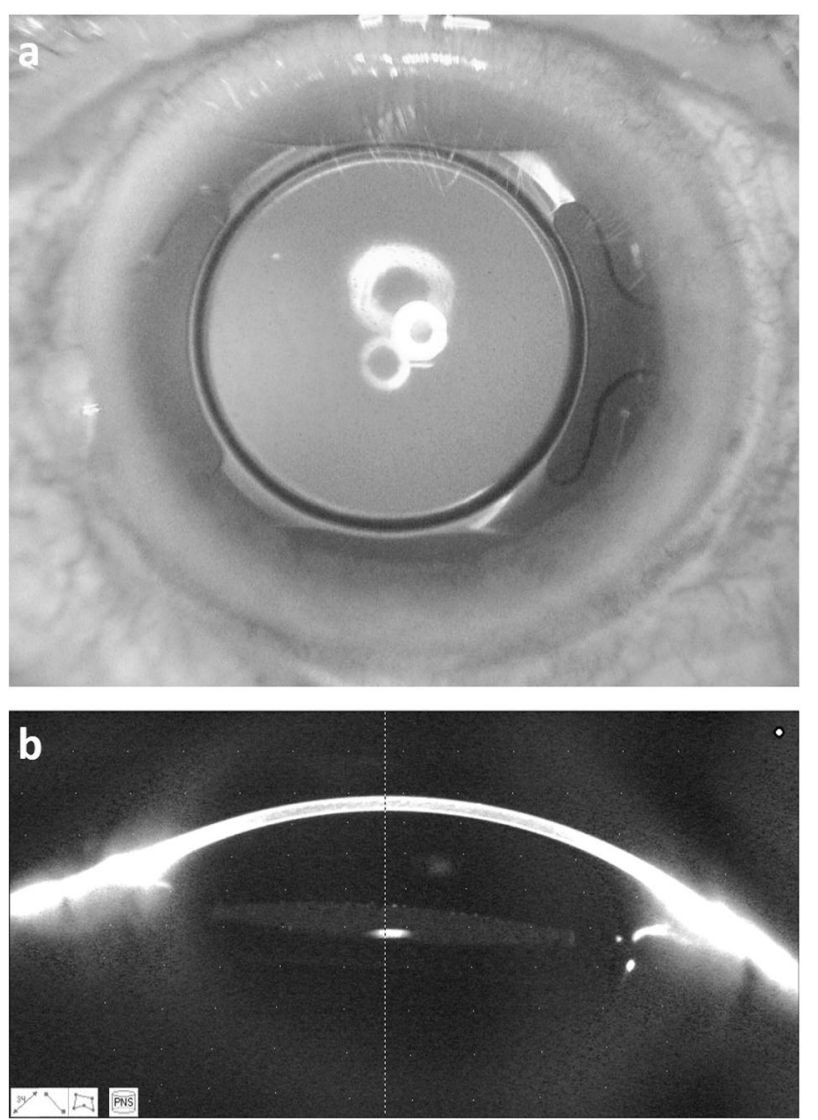

Fig. 2 a Scleral fixation of Akreos AO60 IOL carried out along with 23-gauge pars plana vitrectomy in case 3, a 73-year-old male with post-traumatic subluxated cataract, traumatic mydriasis and glaucoma. Maintenance of excellent centration was seen till 12 months postoperatively. b Scheimpflug imaging using Pentacam confirmed perfect centration of the IOL and absence of tilt

capture within the iris, retinal tear and/or detachment, choroidal detachment, endophthalmitis, prolonged sterile intraocular inflammation, IOL decentration, suture erosion and/or breakage were encountered.

\section{Discussion}

Minimizing the incision size is an important goal in SFIOLs. Decreased operating time by omission of the need for suturing decreases inflammation and phototoxicity. There is reduction of postoperative astigmatism and a faster visual rehabilitation. Creation of a small incision maintains superior anterior chamber stability $[5,15]$, avoids intraoperative hypotony, distortion of globe architecture and minimizes complications such as choroidal effusion and suprachoroidal haemorrhage [4].

SFIOL with PPV has been shown to be a safe procedure $[11,12]$. In fact, PPV removes residual vitreous and capsular remnants, decreasing the postoperative risk of CME 
and retinal detachment [11]. However Vote et al., using 100 polypropylene, showed a high rate of suture breakage on longer follow-up [13]. Also, vitrectomised eyes are prone to scleral collapse during periods of hypotony, thus creating a large incision maybe dangerous for these eyes.

In eyes with anterior chamber angle damage or iris defects, SFIOLs remain the first choice over ACIOLs and iris-fixated IOLs [1-3]. Intrascleral haptic tuck addresses the problem of large incisions when a foldable IOL is used [2]; however, it is fraught with technical challenges and several intraoperative complications such as hyphema, haptic breakage and deformed haptics have been encountered. IOL tilt and pupillary capture can occur in addition to haptic breakage and extrusion $[2,16]$.

Scleral fixation of the Akreos AO60 IOL was first reported in two post-vitrectomy eyes [6], followed by nine eyes with aphakia, with a maximum follow-up of 3.4 months [7]. Recently, scleral fixation of the Akreos AO60 IOL or Alcon CZ70BD IOL using Gore-Tex suture was described in 85 eyes. This was a multicentre study with a mean follow-up of 325 days. While no intraoperative complications were noted, transient hypotony was the commonest postoperative complication seen in $9.4 \%$ cases because of incompetence of 23-gauge wounds [8]. Combined 27-gauge PPV and scleral fixation of the Akreos AO60 IOL using Gore-Tex suture, reported in eight eyes with a mean follow-up of 115 days, did not encounter any case of hypotony or sclerotomy site suturing [9]. Terveen et al described scleral fixation of the Akreos AO60 IOL (in 37 eyes) using 9-0 polypropylene and Gore-Tex suture. The surgeries were performed by three surgeons at their respective institutes with a mean follow-up of 6 months. Ocular hypertension was the most common postoperative complication seen in 24\% [10]. The technique by Fass et al [6] is similar to our technique, except the use of scleral pockets as described by Hoffman. While it has been suggested that performing trans-scleral surgery without flaps is better for scleral integrity [17], creation of one-third scleral thickness rectangular flaps left sutureless is a viable alternative when the knot is not buried within the sclera. This decreases chances of suture erosion through the conjunctiva [18].

BCVA improved significantly in all surgical indications and the IOL was correctly positioned in all cases over a mean follow-up of 8.9 months. There were no major intraoperative or postoperative complications and none of the patients required further surgical interventions. This technique was safe and effective in eyes with pre-existing glaucoma, an important consideration in post-traumatic subluxated or dislocated lenses.

Fixing an IOL at two points may cause the IOL to tilt or decentre [19]. The Akreos AO60 IOL with two paired haptics on either side of the optic has a natural build for 4-point fixation. This allows ease of centration and minimal tilt with simple lacing of the prolene suture. It is easily foldable, allowing use of a smaller corneal incision [9]. Being an aberration-free aspheric lens, it may provide superior quality of vision and improved depth of field [20]. Its optical performance is unaffected by decentration or pupil size due to a uniform centre-to-edge power. This is an additional advantage in eyes with traumatic mydriasis. Intermittent pupillary capture of SFIOL is an early complication especially in vitrectomised eyes [11, 13], and this may be avoided with the Akreos AO60 due to the one piece, four loop haptics with 0 degrees angulation. Single-piece IOLs implanted in the region of ciliary sulcus might lead to iris pigment dispersion, inflammation or glaucoma [21]. Akreos AO60 IOL being hydrophilic acrylic incites less inflammation than hydrophobic acrylic [22].

Scheimpflug imaging confirms the position of implanted IOLs three-dimensionally in vivo and can be used to assess IOL decentration and tilt [23]. The postoperative Pentacam analysis done in six of these eyes showed the absence of IOL tilt or decentration in the current study. It also confirmed absence of haptic sulcus contact.

This technique has a similar set of risks and complications as other SFIOL techniques, except that IOL decentration and tilt appear to be reduced. The use of scleral flaps and rotation of the suture knots as described decrease the risk of suture erosion. Late suture breakage with dislocation of the IOL is a rare complication of SFIOLs. Late suture breakage can be alleviated by using Gore-Tex or 9-0 polypropylene (as was used in this study), which is $60 \%$ stronger than 10-0 polypropylene [7]. The Akreos AO60 IOL is soft with no sharp edges on the haptic eyelets, thus decreasing the risk of cutting of suture [8]. A theoretical concern with this technique is that the IOL optical surface may be damaged by folding the IOL with forceps. These surface irregularities might increase the susceptibility of the IOL to bacterial adhesion [24]. In addition, folding the IOL and implantation with forceps may cause suture tangling or corneal endothelial damage [15].

This study is limited by its retrospective nature, a small heterogeneous sample with an intermediate length of follow-up and absence of a control group. Actual values of vertical/horizontal tilt and decentration on Scheimpflug imaging could add further information. Nevertheless, it is the largest series by a single surgeon with the longest follow-up. In conclusion, I have presented a simple and effective technique for small incision, sutured SFIOL in case is requiring concurrent vitrectomy. This technique produced excellent visual outcomes with well centred and stable IOLs with few complications. 


\section{Summary}

\section{What was known before}

- The Akreos AO60-foldable IOL is amenable to fourpoint sutured fixation with insertion through a small incision in aphakic eyes with insufficient capsular support. This approach to secondary IOL placement is safe and efficacious.

\section{What this study adds}

- Trans-scleral 4-point suture fixation of the Akreos AO60 IOL can be performed easily and safely along with concurrent 23-gauge PPV across varying surgical indications, obviating the need for two surgeries.

- Long-term follow-up in a single centre single surgeon setting shows significant improvement in BCVA with a minimal complication profile.

- Scheimpflug imaging can be used to confirm the position of the implanted SFIOLs 3-dimensionally in vivo.

- This technique is safe and effective in eyes with preexisting glaucoma and traumatic mydriasis.

\section{Compliance with ethical standards}

Conflict of interest The authors declare that they have no competing interests.

\section{References}

1. Por YM, Lavin MJ. Techniques of intraocular lens suspension in the absence of capsular/zonular support. Surv Ophthalmol. 2005;50:429-62.

2. Kumar DA, Agarwal A. Glued intraocular lens: a major review on surgical technique and results. Curr Opin Ophthalmol. 2013;24:21-9.

3. Holt DG, Young J, Stagg B, Ambati BK. Anterior chamber intraocular lens, sutured posterior chamber intraocular lens, or glued intraocular lens: where do we stand? Curr Opin Ophthalmol. 2012;23:62-7.

4. Kay MD, Epstein RJ, Torczynski E. Histopathology of acute intraoperative suprachoroidal hemorrhage associated with transscleral intraocular lens fixation. J Cataract Refract Surg. 1993;19:83-7.

5. Yepez JB, de Yepez JC, Valero A, Arevalo JF. Surgical technique for transscleral fixation of a foldable posterior chamber intraocular lens. Ophthalmic Surg Lasers Imaging. 2006;37:247-50.

6. Fass ON, Herman WK. Sutured intraocular lens placement in aphakic post-vitrectomy eyes via small-incision surgery. J Cataract Refract Surg. 2009;35:1492-7.

7. Fass ON, Herman WK. Four-point suture scleral fixation of a hydrophilic acrylic IOL in aphakic eyes with insufficient capsule support. J Cataract Refract Surg. 2010;36:991-6.
8. Khan MA, Gupta OP, Smith RG, et al. Scleral-fixation of intraocular lenses using gore-tex suture: clinical outcomes and safety profile. Br J Ophthalmol. 2016;100:638-43.

9. Khan MA, Rahimy E, Gupta OP, Hsu J. Combined 27-gauge pars plana vitrectomy and scleral fixation of an akreos AO60 intraocular lens using gore-tex suture. Retina. 2016;36:1602-4.

10. Terveen DC, Fram NR, Ayres B, Berdahl JP. Small-incision 4point scleral suture fixation of a foldable hydrophilic acrylic intraocular lens in the absence of capsule support. J Cataract Refract Surg. 2016;42:211-6.

11. Johnston RL, Charteris DG, Horgan SE, Cooling RJ. Combined pars plana vitrectomy and sutured posterior chamber implant. Arch Ophthalmol. 2000;118:905-10.

12. Bading G, Hillenkamp J, Sachs HG, Gabel VP, Framme C. Longterm safety and functional outcome of combined pars plana vitrectomy and scleral-fixated sutured posterior chamber lens implantation. Am J Ophthalmol. 2007;144:371-7.

13. Vote BJ, Tranos P, Bunce C, Charteris DG, Da Cruz L. Long-term outcome of combined pars plana vitrectomy and scleral-fixated sutured posterior chamber intraocular lens implantation. Am J Ophthalmol. 2006;141:308-12.

14. Loya N, Lichter H, Barash D, Goldenberg-Cohen N, Strassmann E, Weinberger D. Posterior chamber intraocular lens implantation after capsular tear: ultrasound biomicroscopy evaluation. J Cataract Refract Surg. 2001;27:1423-7.

15. Can E, Basaran R, Gul A, Birinci H. Scleral fixation of one piece intraocular lens by injector implantation. Indian $\mathrm{J}$ Ophthalmol. 2014;62:857-60.

16. Kumar DA, Agarwal A, Packiyalakshmi S, Jacob S, Agarwal A. Complications and visual outcomes after glued foldable intraocular lens implantation in eyes with inadequate capsules. J Cataract Refract Surg. 2013;39:1211-8.

17. Lewis JS. Sulcus fixation without flaps. Ophthalmology. 1993;100:1346-50.

18. Solomon K, Gussler JR, Gussler C, Van Meter WS. Incidence and management of complications of transsclerally sutured posterior chamber lenses. J Cataract Refract Surg. 1993;19:488-93.

19. Hu BV, Shin DH, Gibbs KA, Hong YJ. Implantation of posterior chamber lens in the absence of capsular and zonular support. Arch Ophthalmol. 1988;106:416-20.

20. Johansson B, Sundelin S, Wikberg-Matsson A, Unsbo P, Behndig A. Visual and optical performance of the akreos adapt advanced optics and tecnis Z9000 intraocular lenses: Swedish multicenter study. J Cataract Refract Surg. 2007;33:1565-72.

21. Wagoner MD, Cox TA, Ariyasu RG, Jacobs DS, Karp CL, American Academy of Ophthalmology. Intraocular lens implantation in the absence of capsular support: a report by the American academy of ophthalmology. Ophthalmology. 2003;110:840-59.

22. Abela-Formanek C, Amon M, Schauersberger J, Kruger A, Nepp J, Schild G. Results of hydrophilic acrylic, hydrophobic acrylic, and silicone intraocular lenses in uveitic eyes with cataract; comparison to a control group. J Cataract Refract Surg. 2002;28:1141-52.

23. Kim JS, Shyn KH. Biometry of 3 types of intraocular lenses using Scheimpflug photography. J Cataract Refract Surg. 2001;27:533-6.

24. Mencucci R, Dei R, Danielli D, Susini M, Menchini U. Folding procedure for acrylic intraocular lenses. J Cataract Refract Surg. 2004;30:457-63. 